05;06

\title{
Влияние границ зерен на устойчивость нанокластеров алюминия
}

\author{
(C) А.В. Векман, Б.Ф. Демьянов
}

Алтайский государственный технический университет им. И.И. Ползунова, Барнаул, Россия

E-mail: weckman@list.ru

Поступило в Редакцию 2 ноября 2018 г.

Методами молекулярной динамики проведено исследование устойчивости нанокластеров, окруженных матрицей идеального кристалла. Показано, что кластеры вплоть до размеров $10 \mathrm{~nm}$ являются неустойчивыми и растворяются в матрице.

DOI: 10.21883/PJTF.2019.03.47273.17579

Одним из важных вопросов, связанных с нанокристаллическим состоянием металлов, является устойчивость зерен. В настоящей работе предпринята попытка изучения данного вопроса с использованием компьютерной модели, описанной в [1]. Исследовались нанокластеры, окруженные металлической матрицей - идеальным кристаллом из того же материала, что и кластер. Кластер представлял собой усеченный кубооктаэдр, полученный путем отсечения у куба ребер плоскостями типа $\{110\}$ и углов плоскостями типа $\{111\}$ (рис. 1). В результате кластер имел форму многогранника, ограниченного наиболее плотно упакованными кристаллографическими плоскостями типа $\{100\},\{110\}$ и $\{111\}$. Параметр $d$, характеризующий его линейные размеры, задавался при моделировании кластера.

После формирования кластер поворачивали относительно геометрического центра на азимутальный $(\varphi)$ и радиальный $(\theta)$ углы, после чего окружали матрицей. При этом кристаллографические направления решетки матрицы соответствовали кристаллографическим направлениям, изображенным на рис. 1. Следовательно, границы раздела кластер-матрица являются плоскими границами зерен (ГЗ) смешанного типа [1]. Расчеты проводились с использованием парного потенциала Морзе. Параметры потенциала соответствовали алюминию и были взяты из работы [2].

Как показано в [1], усредненные по ансамблю углов значения энергии границ кластер-матрица практически не зависят от размера кластера. Исключение составляют кластеры самых маленьких размеров (<1 nm). Данный результат согласуется с полученным при исследовании индивидуальных плоских границ наклона [3-5]: энергия большеугловых ГЗ слабо зависит как от их вида, так и от угла разориентации.

Исследование поведения нанокластеров, окруженных матрицей, проводилось методом классической молекулярной динамики. Размеры кластеров $d$ варьировались в пределах от 2 до $8 \mathrm{~nm}$. Для каждого размера $d$ было выбрано по восемь комбинаций азимутального и радиального углов поворота ( $\varphi$ и $\theta)$ : 10-0, 20-0, $30-0,40-0,10-10,20-20,30-30,40-40$. Кластеры выдерживались при постоянной температуре $600 \mathrm{~K}$ в течение 10000 шагов. Один шаг интегрирования по времени движения частиц в методе молекулярной динамики был равен 0.01 ps. Таким образом, общее время эксперимента равнялось 100 ps. Использовались жесткие граничные условия. Удаленность закрепленных атомов от границы кластер-матрица составляла более пяти параметров решетки. Это позволяет утверждать, что закрепленные атомы не оказывают силового воздействия на атомы, находящиеся вблизи границы. В процессе работы компьютерной программы отслеживалось диффузионное движение атомов, создавался видеофайл перемещения атомов в сечении, соответствовавшем середине кластера, и производилась регистрация потенциальной и кинетической энергии, приходившейся в среднем на один атом расчетной ячейки.

Расчеты показали, что все кластеры „растворяются“ в матрице, при этом система эволюционировала к состоянию с минимумом потенциальной энергии. Поэтому главной задачей было определить по полученным данным, в какой момент происходит растворение кластера в зависимости от его параметров (размера $d$, азимутального $\varphi$ и радиального $\theta$ углов поворота относительно матрицы).

В процессе проведения расчетов было замечено, что диффузионное движение атомов происходит только на границе кластер-матрица. Данное обстоятельство обусловлено наличием большого количества структурных вакансий на границе. Эти вакансии создают избыточный объем, который в значительной степени оказывает влияние на поведение системы. Расчет значения избыточного объема является непростой задачей, так как зависит
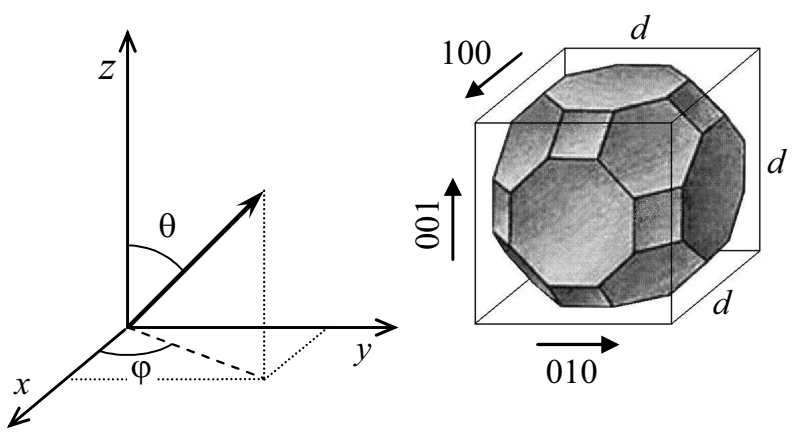

Рис. 1. Внешний вид моделируемого кристалла. 


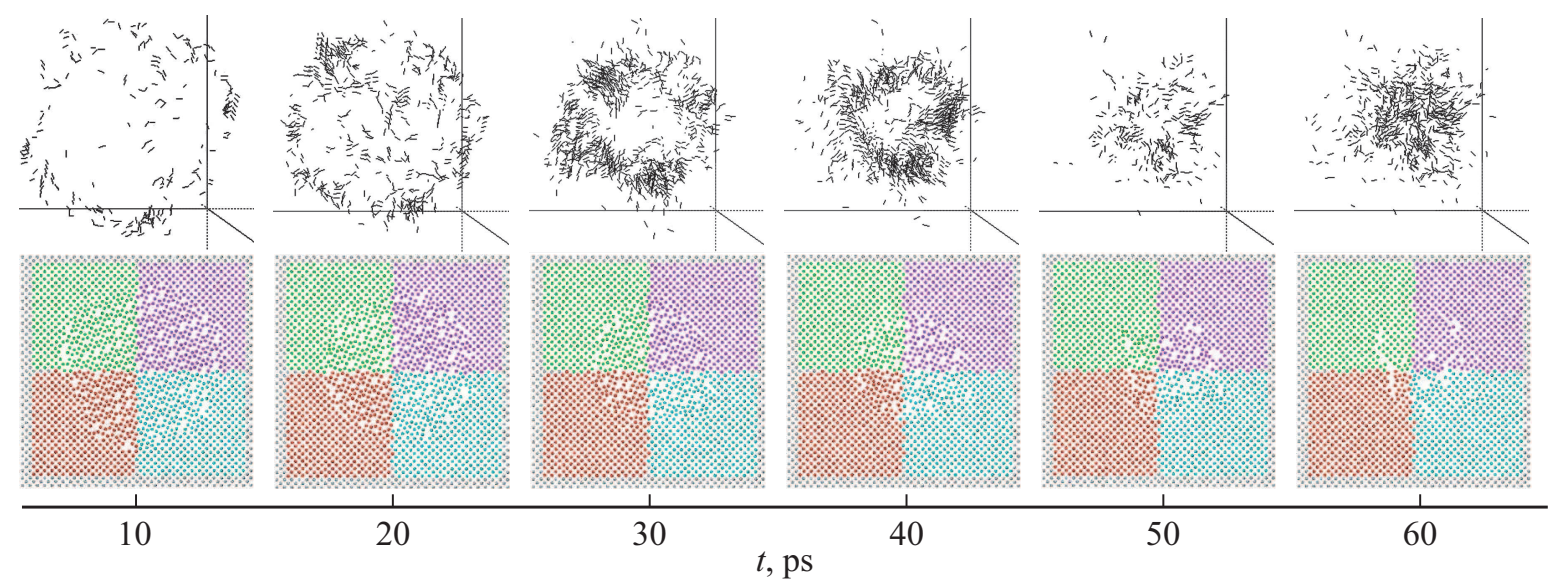

Pис. 2. Динамика диффузионного движения атомов и кадры растворения кластера в процессе релаксации.
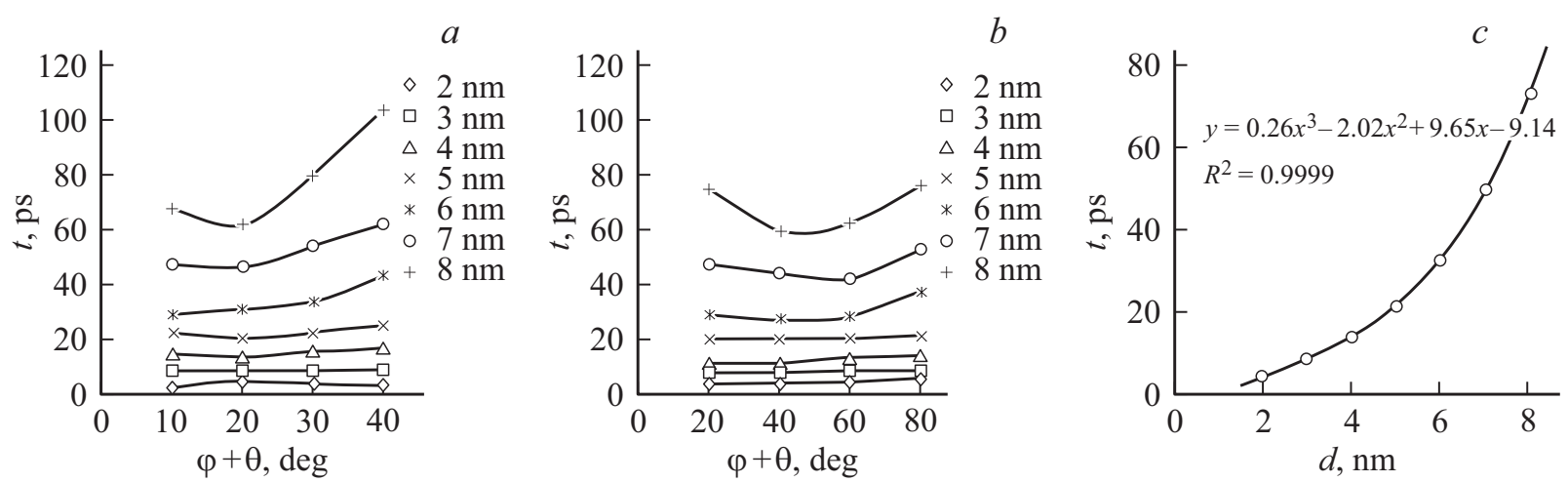

Рис. 3. Зависимости времени растворения кластера от ориентации относительно матрицы с нулевым радиальным углом $(a)$, с ненулевым радиальным углом $(b)$ и от размера кластера $(c)$.

от того, какую область кристалла используют для его определения. В нашем случае логично было выбрать шаровой слой в области границы кластер-матрица. Поскольку границы являются плоскими ГЗ, а их толщина составляет по разным данным не менее двух параметров решетки, было принято решение взять толщину слоя, равную четырем параметрам кристаллической решетки: по два в каждом направлении от границы. В таблице приведены значения избыточного объема исследованных кластеров.

Видно, что при таком расчете свободный объем составляет от 2 до $6 \%$, что, вообще говоря, достаточно много. Однако количество вакансий, рассчитанное на весь расчетный блок, не превышает $1.5 \%$ от общего числа атомов. Кроме того, не наблюдается зависимости значений избыточного объема ни от ориентации кластера, ни от его размеров. Этот результат согласуется с полученным при исследовании ГЗ [6], избыточный объем которых не зависит ни от их вида, ни от угла разориентации.

Динамика диффузионного движения атомов кластера с размером $d=8 \mathrm{~nm}$ и углами поворота $\varphi=20^{\circ}$ и $\theta=20^{\circ}$ представлена на рис. 2. Из рисунка видно, что
Свободный объем границы кластер-матрица в расчете на объем шарового слоя, содержащего границу

\begin{tabular}{c|c|c|c|c|c|c|c|c}
\hline \multirow{2}{*}{$\varphi,^{\circ}$} & \multirow{2}{*}{$\theta,{ }^{\circ}$} & \multicolumn{7}{|c}{ Размер кластера $d$} \\
\cline { 3 - 9 } & & $2 \mathrm{~nm}$ & $3 \mathrm{~nm}$ & $4 \mathrm{~nm}$ & $5 \mathrm{~nm}$ & $6 \mathrm{~nm}$ & $7 \mathrm{~nm}$ & $8 \mathrm{~nm}$ \\
\hline 10 & 0 & 0.036 & 0.036 & 0.034 & 0.046 & 0.042 & 0.040 & 0.044 \\
20 & 0 & 0.043 & 0.039 & 0.043 & 0.031 & 0.041 & 0.043 & 0.039 \\
30 & 0 & 0.021 & 0.032 & 0.049 & 0.040 & 0.047 & 0.039 & 0.039 \\
40 & 0 & 0.029 & 0.030 & 0.042 & 0.043 & 0.038 & 0.040 & 0.040 \\
10 & 10 & 0.053 & 0.058 & 0.058 & 0.054 & 0.060 & 0.056 & 0.058 \\
20 & 20 & 0.052 & 0.050 & 0.053 & 0.056 & 0.054 & 0.054 & 0.054 \\
30 & 30 & 0.050 & 0.049 & 0.057 & 0.056 & 0.064 & 0.057 & 0.064 \\
40 & 40 & 0.049 & 0.048 & 0.056 & 0.056 & 0.059 & 0.056 & 0.056
\end{tabular}

в начале эксперимента $(t<40 \mathrm{ps})$ диффузия идет только по границе кластер-матрица. В процессе релаксации эта область уменьшается, причем диффузионное движение в середине кластера отсутствует. Уменьшение размеров кластера не сопровождается изменением внутренней структуры кластера. В момент времени $t=50 \mathrm{ps}$ в центре кластера остается область порядка 2-3 параметров решетки, в которой также нет перескоков атомов. На 
этом этапе кластер практически аннигилировал. И наконец, при времени эксперимента 60 ps кластер становится настолько малым, что диффузионное движение занимает весь его объем. На этом этапе можно считать, что весь кластер растворился в матрице.

После обобщения полученных экспериментальных данных по диффузионному движению атомов и видеоряду нами были установлены закономерности времени растворения кластера в матрице. Эти результаты отражены на рис. 3.

На рис. 3, a представлена зависимость времени растворения кластера с нулевым радиальным углом $\theta$. Для кластеров с размерами $5 \mathrm{~nm}$ и менее зависимость времени растворения от их ориентации относительно матрицы отсутствует. Для кластеров с размерами $6 \mathrm{~nm}$ и более появляется зависимость от угла разворота. С увеличением $\varphi$ время растворения имеет тенденцию к росту, причем рост не является линейным.

На рис. 3, $b$ изображены зависимости времени растворения кластера с ненулевым радиальным углом $\theta$. Из рисунка видно, что для кластеров с размером $5 \mathrm{~nm}$ и менее отсутствует зависимость времени растворения от их ориентации относительно матрицы. Кластеры больших размеров растворяются за разное время, но какой-либо тенденции влияния ориентации в матрице не наблюдается.

Из рис. 3, $a$ и $b$ также видно, что время аннигиляции кластеров растет с увеличением их размера. Это объясняется тем, что диффузионный процесс идет только по границе кластер-матрица. В связи с этим представляет интерес выяснение зависимости среднего времени аннигиляции от размеров кластера. Эта зависимость приведена на рис. 3,c. Видно, что она носит явный степенной характер. Параметры аппроксимации и величина ее достоверности $R^{2}$ отражены на рисунке и позволяют оценить время аннигиляции для кластеров бо́льших размеров, чем исследованные в настоящей работе. Можно ожидать, что нанозерна алюминия, окруженные матрицей, станут устойчивыми, если их размеры превысят значение $10 \mathrm{~nm}$.

\section{Список литературы}

[1] Векман А.В., Адарич Н.В., Драгунов А.С., Демьянов Б.Ф., Агейкова Л.Н. // Фундаментальные проблемы современного материаловедения. 2011. Т. 8. № 2. С. 24-27.

[2] Царегородиев А.И., Горлов Н.В., Демьянов Б.Ф., Старостенков М.Д. // ФММ. 1984. Т. 58. № 2. С. 336-343.

[3] Rittner J.D., Seidman D.N. // Phys. Rev. B. 1996. V. 54. N 10. P. 6999-7015.

[4] Tschopp M.A., Mcdowell D.L. // Phil. Mag. 2007. V. 87. N 25. P. 3871-3892.

[5] Homer E.R., Foiles S.M., Holm E.A., Olmsted D.L. // Acta Mater. 2013. V. 61. N 4. P. $1048-1060$.

[6] Векман A.B. Атомная структура и энергия границ зерен наклона типа $[100]$ в кубических кристаллах. Канд. дис. Барнаул: Алт. гос. техн. ун-т им. И.И. Ползунова, 2000. $182 \mathrm{c}$. 